\title{
Universidades salvadoreñas
}

\section{en la sociedad de la información \\ y el conocimiento con \\ perspectivas de género}

Camila Calles Minero camila.calles@utec.edu.sv

Investigadora UTEC

Sabemos muy poco, y sin embargo es sorprendente que sepamos tanto, y es todavía más sorprendente que tan poco conocimiento nos dé tanto poder

Bertrand Russell

\section{RESUMEN}

El mundo entero experimenta una revolución. La información y el conocimiento son la base productiva en la actualidad, por ende la generación del conocimiento y la adecuada gestión de la información son elementales para no quedar al final de la fila entre las sociedades que avanzan. Las universidades salvadoreñas, ¿qué hacen al respecto? ¿Cómo gestionan la información? Esta investigación pretende mostrar cómo en El Salvador se gestiona la sociedad de la información y del conocimiento, vista desde las universidades, muy específicamente desde los niveles de manejo de la información para generar conocimiento en las instituciones de educación superior. Para ello se entrevistó a personeros de las universidades, a quienes preguntó sobre el flujo de información en las instituciones, y sobre el manejo de los páginas Web, entendiendo estas últimas como el medio de comunicación de la institución para mostrar su quehacer y el contacto con sus usuarios. La investigación contempló además, el análisis de las páginas Web, con la herramienta T-incluye, la cual analiza el uso de sintagmas no incluyentes, es decir, aquellos en donde las mujeres quedan invisibilizadas. Se indagó sobre la presencia de políticas institucionales sobre la equidad de género y las iniciativas que existen dentro de las instituciones de educación superior sobre el uso de lenguaje inclusivo en el flujo de datos que en ellas se produce, así como en la divulgación de los mismos.

Palabras claves: Sociedad de la información y del conocimiento, universidades, comunicación, páginas electrónicas, digitalización de la información, lenguaje de género, lenguaje inclusivo. 


\section{ABSTRACT}

The world experiences a revolution. The information and knowledge are currently the productive base, thus the generation of knowledge and the appropriate management of the information are basic to keep away from the end of the row between societies advancing. Wath do Salvadorean universities do in this respect? How do they manage the information? This research aims to show how in El Salvador the society of information and knowledge, is managed in universities, specifically from the levels of information management to generate knowledge brewed within higher education institutions. To do so, were interviewed representatives of the universities where he wondered about the flow of information in the institutions, and the management of Web pages, understanding the latter as a mean of communication of the institution to show their work and contact with users. Research also included analysis of Web pages, with the T-includes tool, which analyzes the use of not inclusive phrases, those where women remain invisible, asked about the presence of institutional policies on gender equity and initiatives that exist within the institutions of higher education on the use of inclusive language in the data flow that occurs, as well the dissemination of the same.

Keywords: society of information and knowledge, universities, communication, electronic pages, digitalization of information, gender, inclusive language.

\section{Introducción}

D e empujar el arado sobre la tierra hasta cultivar a pulsar las teclas de una computadora para producir difundir y recibir información, es el camino recorrido por las sociedades de todo el mundo en busca de generar ganancias y poder, tanto económico como social y político. El Salvador no se escapa de ello, y por supuesto, las universidades del país están envueltas en esa realidad.

Es evidente que hoy en día el mundo entero está transformándose, una nueva revolución se gesta, un cambio en donde la información y el conocimiento son la base y sustancia del sistema productivo y social. El conocimiento vale por el poder que éste genera al ser creador de tecnología, de ciencia, de políticas o de cualquier elemento que permite cambios sustanciales en la sociedad.
Esta nueva era que viven las sociedades actuales y específicamente las universidades, está caracterizada no solo por el papel protagónico que tienen el conocimiento y la información, sino además, por la búsqueda de la forma de aplicación del conocimiento y la información para generar conocimiento, además del procesamiento de la información y comunicación, en un círculo de retroalimentación acumulativo entre la innovación y sus usos (Castells, 2001a).

Por su parte, Cazaux (2010) establece que la noción de sociedad del conocimiento surgió hacia finales de los años 90 y es empleada particularmente en medios académicos, como alternativa a la sociedad de la información. La sociedad del conocimiento presume un paso delante de la sociedad de la información, un paso en donde los simples datos e información pura sirven para producir conocimiento, que a la vez es gestionado para poder ser utilizado de manera productiva. 
Unesco (2005) adoptó el término sociedad del conocimiento, y la caracteriza por las transformaciones producidas por la acelerada introducción en la sociedad de la inteligencia artificial y de las tecnologías de la información y la comunicación (TIC), de acuerdo con Cazaux (2010), quien además dice que la Organización de Estados Iberoamericanos (OEI), al igual que el conjunto de sociedades mundiales, reconocen que se encuentran en un nuevo marco de actuación sustentado especialmente, en las TIC y en la biotecnología.

En ese contexto las sociedades tienen que construir nuevos escenarios de actuación que les permitan sacar el mayor provecho a la producción de conocimiento mundial, pues de lo contrario éste será recurso desperdiciado. Se tiene que realizar una efectiva gestión del conocimiento, que signifique saber cómo acceder a las informaciones, seleccionar esa información, articularla y aplicarla a un determinado objetivo. (Fontcuberta, 2000).

Según la Comisión Económica para América Latina y el Caribe (Cepal) el concepto de sociedad de la información fue creado por Machlup (1962, citado por Cepal, 2008), cuya conclusión fue que el número de personas dedicadas al manejo y procesamiento de información es mayor que el de quienes realizaban tareas físicas. (Cepal, 2008).

Otros autores, como Drucker (1969, citado por Cepal 2008) y Bell (1973, citado por Cepal 1999), auguraron que el conocimiento es el principal factor de generación de riqueza en la sociedad. Por su parte, Masuda (1981, citado por Cepal 2008) analizó el papel de la información y establece que es el principal elemento en el proceso actual en donde prevalecen las condiciones tecnológicas.

Castells (2001b) afirma que el proceso actual de transformación tecnológica se expande de forma exponencial, por todo el mundo, por su capacidad para crear una interfaz entre los campos tecnológicos mediante un lenguaje digital común en el que la información se genera, se almacena, se recobra, se procesa y se transmite, además se caracteriza por la aplicación de ese conocimiento e información a aparatos de generación de conocimiento y procesamiento de la información/comunicación.

Otro autor que habla de la importancia del conocimiento y la información en las sociedades actuales es Toffler (1992), quien establece que en raros momentos de la historia, el avance del conocimiento ha acometido y traspasado antiguas barreras. "Los más importantes de estos descubrimientos se han relacionado con la invención de nuevas herramientas para el pensamiento y la comunicación, como el ideograma, el alfabeto, el cero y en nuestro siglo el ordenador" (Toffler, 1992, p 114).

Por su parte, Cepal (2005) asegura que el concepto de sociedad de la información hace referencia a un paradigma que está produciendo profundos cambios en el mundo desde el comienzo de este milenio. Esta transformación está impulsada principalmente por los nuevos medios disponibles para crear y divulgar información mediante tecnologías digitales. Los flujos de información, las comunicaciones y los mecanismos de coordinación se están digitalizando en muchos sectores de la sociedad, proceso que se traduce en la aparición progresiva de nuevas formas de organización social y productiva.

La información ha tenido un papel fundamental a través de la historia en el desarrollo de las sociedades y por supuesto de la humanidad, a eso se le suma la posibilidad de compartirla mediante las diferentes formas de comunicación que existen que sigue asombrando a hombres y mujeres. En ese sentido, actualmente el intercambio de información es 
lo que define la conducta de la humanidad en su conjunto. (Hilbert y Cairó, 2009).

Si El Salvador es parte de este mundo global y está inmerso en la sociedad de la información tiene que establecer sus objetivos para alcanzar la sociedad del conocimiento, además de determinar los medios a utilizar para llegar a ello, que debe de estar planeado en conjunto entre todas las estructuras que dinamizan los procesos sociales en el país. Tomando, por supuesto, de referencia los ejemplos internacionales y más inmediatos, pues no se puede ver como una isla en medio del mar de la información, ya que los ciudadanos buscan constantemente difundir su información y necesitan recibir información de su interés. El manejo de las TIC, la digitalización de la información tiene que ser parte de ese camino y las universidades salvadoreñas deben meterse en ello.

Las TIC no sólo son instrumentos tecnológicos de emisión, difusión y recepción de mensajes, ya que el proceso no sólo cuenta de la divulgación de la información, sino que además permiten una comunicación interactiva. Hoy en día vivimos un proceso de fusión de las tecnologías de información y divulgación, las tecnologías de la comunicación y las soluciones informáticas (Cepal, 2005).

El uso de las TIC conlleva realizar un proceso de digitalización de datos. Lo que da entrada a la sociedad del conocimiento no solo es la estructura que tiene, sino el flujo de las comunicaciones ante la existencia de la tecnología. El objetivo es entonces digitalizar los flujos de información y las comunicaciones en diferentes ámbitos de la sociedad, tales como las empresas, el comercio, la atención sanitaria, la administración pública, la educación y otros. (Cepal 2005).
Esos flujos de información se pueden evidenciar a través de las páginas electrónicas, puesto que éstas constituyen un nuevo tipo de producto audiovisual en donde la integración de diferentes lenguajes con igualdad de importancia conjuga mensajes para difundir información. (Valdés, 2008 y Observatorio de la Cibersociedad, 2004).

En ese sentido, este estudio describe cómo gestionan la información y el conocimiento las universidades salvadoreñas, para ello, se identificó cómo generan, articulan y distribuyen el conocimiento; en segundo lugar, se identificó el uso y presencia de TIC en esa gestión; y, en tercer lugar, se identificó si la gestión del conocimiento y de la información en las universidades salvadoreñas se realiza con enfoque de género.

\section{Método}

La investigación es de tipo cualitativo. Se realizaron siete entrevistas a personeros de siete universidades salvadoreñas, sobre elementos del manejo y digitalización de la información que se genera dentro de las instituciones de educación superior. El estudio pretendía realizar entrevistas en las 24 universidades inscritas ante el Ministerio de Educación, sin embargo sólo siete colaboraron. Se observaron 20 portales electrónicos de las universidades, en donde se utilizó la herramienta Web T-incluye, la cual permite analizar el lenguaje incluido en las páginas Web. Esta herramienta fue creada por el Centro Tecnológico de la Información y de la Comunicación (CTIC) quien gestiona el proyecto "Web con Género". EI CTIC promueve y estimula actividades relacionadas con el desarrollo de las TIC en todos los campos de la vida económica y social, que conduzcan al impulso y fortalecimiento de la Sociedad de la Información. (Web con género, 2005). 


\section{Resultados}

Las siete universidades entrevistadas tienen computadoras para uso docentes (tiempo completo), las cuales tienen conexión a Internet, además, todos los docentes tienen la opción de trabajar en red entre ellos (entendiendo red con la opción de trabajar en conjunto algo, no necesariamente con conexión a Internet). También está la presencia de computadoras para uso de estudiantes, de éstas sólo el 85 por ciento tiene conexión a Internet.

No todas las universidades estudiadas tienen Internet inalámbrico en su campus. Todas las universidades tienen portal electrónico institucional, a través del cual publican información general. El 60\% de las universidades administran la información de los portales únicamente a través del departamento de informática. Un 25\% lo hace sólo el departamento de comunicaciones o relaciones públicas. El 15\% lo hacen en conjunto informática con relaciones públicas.

El $80 \%$ de las universidades investigadas no tienen un período específico para la actualización de la información que se publica en la página electrónica. La información que se publica en la página Web se obtiene directamente de los departamentos generadores de información (administración académica, los decanatos, nuevo ingreso, entre otras). La información que se publica en algunos casos es aprobada por la administración general de las universidades, en otros, las unidades específicas que quieren publicar información nueva producen la información y la trasladan directamente al departamento de informática o comunicaciones, la información que se publica es revisada en cuanto a ortografía y gramática, el contenido depende de los generadores de la información, se revisa el arte gráfico, este último proceso, en algunas ocasiones lo hacen en conjunto los departamentos de informática y comunicaciones.
No todas las universidades salvadoreñas realizan conteo de visitas y uso del portal electrónico. Solo una de las siete entrevistadas lo realiza. Igualmente, en ninguna de las páginas Web se detectó la opción de ver el contenido en inglés u otro idioma, sólo en español.

El nivel de digitalización de las universidades lo domina la información estudiantil, es la que tiene mayor nivel de información ya digitalizada; en segundo lugar, la información académica (sobre planes de estudio, carreras, materias); y en tercero la administrativa (procesos dentro de la universidad). La digitalización de la información dentro de las universidades la realiza cada unidad que produce esa información, en algunos casos, los departamentos de informática.

No todas las universidades tienen sus producciones científicas digitalizadas. Tres de las universidades investigadas no tienen revistas especializadas como medio de divulgación; cuatro si tienen revistas pero sólo dos de ellas tienen versión digital; En cuanto a los trabajos de graduación, sólo una de las siete universidades estudiadas no los tienen digitalizados. Las producciones científicas y estudiantiles no se divulgan en su totalidad a través de la página Web.

Todas las universidades estudiadas poseen correo electrónico institucional. De manera interna utilizan con mayor frecuencia el papel (memorándum, cartas, solicitudes por escrito); en la comunicación externa utilizan el correo electrónico (invitaciones de actividades de las instituciones y avisos institucionales, correspondencia al extranjero). Las universidades aún no prestan el servicio de la realización de trámites estudiantiles de manera virtual, sólo dos de las universidades tiene opciones virtuales.

Solo en cuatro de las siete universidades entrevistadas se pueden hacer consultas virtuales a sus bibliotecas, de esas 
cuatro, en tres se pueden ver títulos y libros, en la otra sólo títulos. Solo una de las universidades no está en red con bibliotecas o bases de datos extranjeras.

El $30 \%$ de las universidades entrevistadas imparten carreras virtuales y el mismo porcentaje es para las que tienen asignaturas semipresenciales. En las asignaturas que imparten, de manera presencial, sólo el 10 \% tienen de manera institucional la posibilidad de hacer uso de espacios virtuales de apoyo.

Ninguna de las universidades estudiadas tiene políticas institucionales en el tema de género. Una de las universidades tiene como eje transversal en sus planes de estudio la equidad de género, pero asumido dentro de valores humanos y cristianos que la institución, no específicamente bajo la teoría de género. Solo dos universidades tienen alguna iniciativa para promover la equidad de género al interior del recinto académico, otra universidad tiene en proceso la creación de un programa con esa naturaleza. Ninguna de las instituciones regula el uso del lenguaje sexista en el portal electrónico, en la comunicación interna y en los trabajos de graduación de su universidad. De los 20 portales analizados con la herramienta T-incluye, en todos se encontró la presencia de lenguaje no inclusivo, los 20 sintagmas que denotan la invisibilidad de las mujeres más frecuentes son: Los estudios(110), de los estudiantes(105), los alumnos(102), nuestros estudiantes(90), nuestros docentes(90), un profesional(89), el profesional(88), nuestros egresados(75), los docentes(74), del nuevo profesional(72), nuestro profesional(70), los maestros(67), los documentos(65), bienvenidos(64), los científicos(62), usuario(61), hombres(60), nuestros universitarios(59), todos(58), el rol(40).

\section{Discusión}

Todas las universidades salvadoreñas tienen computadoras para el uso del personal docentes a tiempo completo, sin embargo, las instituciones pequeñas no tienen equipo destinado para los docentes hora clase. Los docentes de planta de las universidades en donde se realizó la entrevista, tienen la opción de trabajar en red (no necesariamente con conexión a Internet), sin embargo, hasta donde se pudo indagar esa conexión en red es utilizada para aspectos técnicos, como a la hora que los departamentos de informática realicen trasferencias de documentos, pero para el traslado de información o el trabajo de información conjunta los docentes no la utilizan.

Sólo el 85 \% de las computadoras a disposición de los estudiantes tienen conexión a Internet, es decir que no todos los estudiantes pueden conectarse al Internet, la posibilidad de investigar, inspeccionar en el espacio virtual se estrecha, en una sociedad en donde es elemental; la capacidad de viajar por el mundo virtual se reduce aún más, pues no todas las universidades salvadoreñas tienen conexión a Internet de manera inalámbrica.

Un punto a favor de todas las universidades de El Salvador es que tienen portal electrónico, sin embargo estos no funcionan al $100 \%$, cuando se visitó los diferentes links de cada portales en un $25 \%$ no se logró ingresar, por dos razones básicas: no tenían la información que anunciaba en su etiqueta y por el contrario repetían información de la portada, y por fallas en el servidor, al menos en el momento de la realización (diciembre-2010 / enero, febrero y marzo 2011). 
Las páginas electrónicas son utilizadas como herramientas de difusión de la información básica de las universidades, en algunas se colocan enlaces para otras páginas institucionales, como las bibliotecas o los medios de comunicación que poseen. La administración de la información que se publica en la página Web de las universidades es muy técnica dado que es manejada solamente por un solo departamento, o solo informática o solo relaciones públicas, en los casos que lo hacen de manera conjunta, se pude ver en los portales mayor fluidez de la información y mejor manejo de los datos, pues es un trabajo conjunto.

En la sociedad de la información y del conocimiento, un elemento importante e indispensable es la utilización de información digital a través del Internet, incluso el manejo de datos es ya una forma de organización en la que el uso eficiente de la información digital por medio de las TIC permite optimizar procesos, generando un mayor valor económico y social con efectos positivos para el desarrollo de los países. Cepal (2008).

En las universidades que se investigaron no existe un tiempo establecido para actualizar la información de los portales. La mayoría de la información es estática, permanentemente sirve de la misma manera, pues es información básica de la universidad. Muy pocos portales universitarios tienen la difusión de información dinámica. Las páginas Web podrían servir para difundir información generada por las actividades diarias de la universidad. Actualmente, las páginas electrónicas se alimentan de datos generados por las diferentes unidades, en los casos en donde la administra el departamento de informática no recibe ningún tratamiento para su publicación, es decir que son publicadas tal cual las envían. La información debiera recibir criterios comunicacionales de publicación. La revisión que tiene es únicamente de diseño gráfico, ortografía y gramática.
Las páginas electrónicas de las universidades son un medio de comunicación y tienen que ser considerados como tal por las instituciones educativas, para ser utilizadas de la mejor manera y poder hacer buen uso de la información que ahí se publique. En esta nueva era de la sociedad, en donde la información y el conocimiento son los pilares, los medios de comunicación y la digitalización de la información se moldean a esa realidad y con ello tiene que cambiar la forma en que el conocimiento se administra. Fontcuberta (2000) propone la aparición de la idea de gestión del conocimiento, entendida en el sentido de saber cómo acceder a las informaciones necesarias, seleccionarlas, articularlas y aplicarlas a un determinado objetivo.

La información digital que las universidades tienen aún es muy pobre, hace falta poseer más información digital del área administrativa sobre todo. La digitalización de la información en las universidades salvadoreñas varía, en algunas instituciones los realiza la propia unidad de donde emana la información, en otras las personas del departamento de comunicaciones son quienes levantan el texto digital, de acuerdo a las necesidades comunicacionales, y en otras universidades el encargado es el departamento de informática.

Cepal (2008) asegura que la digitalización de datos por medio de las TIC tuvo profundas repercusiones en la generación, almacenamiento, procesamiento, intercambio y difusión de la información, transformándola en un recurso esencial en toda actividad económica y social. Ahora es un elemento importante e indispensable dentro de la sociedad de la información, incluso es ya una forma de organización en la que el uso eficiente de la información digital por medio de las TIC permite optimizar procesos, generando un mayor valor económico y social con efectos positivos para el desarrollo de los países. 
Todas las universidades que se entrevistaron afirmaron poseer correo electrónico institucional, sin embargo, para efectos de esta investigación las comunicaciones con muchas universidades fue a través de correos personales. En las comunicaciones internas, las universidades utilizan con mayor frecuencia el papel. El correo institucional es más para comunicación. Hay que evidenciar que en algunas de las universidades en donde se realizó la entrevista, el flujo de comunicación interna a través del correo electrónico cada vez crece.

El sistema presencial para la realización de trámites por parte de los estudiantes aún prevalece, los estudiantes tienen que ir a las instalaciones de las universidades para gestionar su ingreso, la inscripción de asignaturas, la consulta de notas, etcétera. Muy pocas universidades se han dado a la tarea de gestar esas acciones para que se realice únicamente por la vía digital o de ambas forma.

En ese sentido, Conacyt (2010) establece que en el país el grado de implementación de las TIC en las instituciones de educación superior es bastante amplio, sin embargo, aún está pendiente la utilización del extranet, es decir que los estudiantes tengan la posibilidad de gestionar sus demandas y servicios desde fuera del campus a través de internet.

Las universidades salvadoreñas utilizan herramientas Web como el Internet, el correo electrónico y la página Web, según Conacyt (2010) éstos son los recursos más utilizados. En cuanto a las TIC, de igual manera, la conectividad interna de las instituciones educativas se encuentra en un $72.97 \%$ y el $86.49 \%$ de las universidades tienen Internet inalámbrico en sus instalaciones. El $54.05 \%$ de las instituciones cuentan con un sistema informático al que se puede acceder desde lugares fuera de la institución.
Según el estudio realizado en siete universidades salvadoreñas, se detectó además que no todas las producciones científicas de las universidades están digitalizadas, igualmente no todas poseen un medio de divulgación escrito y que se puede acceder de manera digital, como revistas especializadas. En cuanto a los trabajos y producciones estudiantiles como trabajos de graduación, la mayoría de las universidades si los tiene digitalizado.

Una de las ventajas de la digitalización, según el Museo de la información, es que a través de ella se pueden buscar datos específicos en volúmenes muy grandes de información. El acceso a bibliotecas mundiales, a bases de datos específicas por áreas del conocimiento, contenidos de libros completos que están al alcance de muchas personas.

No en todas las bibliotecas de las universidades salvadoreñas estudiadas se puede hacer consultas electrónicas, y en las que se puede, solo se tiene la opción de ver títulos, en muy pocas de títulos y libros. Eso restringe el acceso a los libros por parte de estudiantes y docentes. Lo mismo sucede con los trabajos de graduación, los cuales no pueden ser consultados de manera virtual.

De acuerdo con Murolo, (2010), a través de la digitalización de datos, la sociedad transmite la información y el conocimiento con un formato digital, si antes había que ir a la biblioteca a consultar un libro, hoy se tendría que poder sin salir de la casa. Varias bibliotecas en el mundo están digitalizando sus libros para que puedan ser consultados por Internet. En un futuro cercano, y gracias a la digitalización, los programas de radio y televisión también podrán estar en la esfera digital, así como la posibilidad de acceder a los archivos de televisoras y radiodifusoras en Internet para rentar algún programa, o tener a la mano bases de datos. 
Según el estudio realizado por Conacyt en el 2009, el $80 \%$ de las universidades en El Salvador tiene posibilidad de ingreso a bibliotecas virtuales, el $49 \%$ tiene plataformas para el desarrollo de educación a distancia, o educación virtual, el $41 \%$ de las instituciones transmiten información a través del Internet y sólo el 9 \% se puede realizar gestión administrativa o académica vía Internet. (Conacyt, 2010).

(Conacyt detalla que en El Salvador el Internet es utilizado en un 100\% para envío y recepción de correo electrónico.) El $80 \%$ de las instituciones hace uso del Internet para búsqueda de información a bibliotecas virtuales, esos porcentajes han aumentado del 2008 al 2009. Sin embargo, en cuanto al uso de Internet para descarga de impresos o formularios tiene un 13\%\% para el 2009 y Gestión administrativa y/o académica electrónica el 25\%, eso no significó crecimiento con relación al 2008.

Conacyt (2010) establece que las TIC cada vez se propagan más en las instituciones de educación superior con únicamente tres limitantes: la primera es que el acceso externo a la información y gestión administrativa de las instituciones desde internet es reducida; en segundo lugar, hay poco uso de la video conferencia como recurso de educación a distancia y; falta cobertura del uso de las TIC en las instalaciones de las universidades, es decir, poca utilización del Internet inalámbrico.

De las siete universidades en las que se pudo realizar las entrevistas, la mayoría están adscritas a redes avanzadas, eso significa que están en contacto con otras instituciones de educación superior, nacional e internacional y tienen alianzas con otras universidades, centros científicos y centros de documentación. Las instituciones salvadoreñas no están aisladas de lo que está pasando en el mundo, están en alian- zas con otras para realizar trabajos de investigación e incluso académicos, hay universidades que tienen programas conjuntos con universidades europeas. Estas alianzas muy pocas universidades las dejan ver en sus portales electrónicos, más bien es información que solo se maneja a nivel interno.

El hecho que las universidades aún no contemplan el medio de comunicación digital como válido para la trasferencia de información universidad-estudiantes, a eso se suma que los estudiantes tienen poco acceso a una computadora con conexión a internet y por ello las universidades no pueden hacer más uso de la información digital y sacarle más provecho al dinamismo que genera una información digital contra la tradicional forma de comunicación en papel.

Esta información digitalizada necesita de un medio para ser difundida, para poder ser trasladada del emisor a los receptores, y ese medio es el Internet (que hace un símil con los papeles impresos de Gutenberg, al extender el conocimiento). Por medio de Internet la información digital puede difundirse, el conocimiento digital puede ser consumido cada vez por más personas; sin embargo surge el tema de la brecha digital, que es hasta ahora el mayor obstáculo que no le permite a la información digital desarrollarse y expandirse.

En El Salvador hace falta el diseño de políticas públicas orientadas exclusivamente a reducir la brecha digital y establecer los cimientos de la sociedad de la información. Lemus y Villatoro (2009) retoman datos del Banco Mundial en donde se establecen que la brecha digital en cuanto al acceso a computadoras y al uso de Internet aún es grande entre los países desarrollados y los países en vías de desarrollo, afirmación que se justifica con el número promedio de teléfonos, computadoras y usuarios de Internet en América Latina 
y El Salvador comparadas con las del grupo de países conocidos como G7 (Alemania, Canadá, Estados Unidos, Francia, Italia, Japón y el Reino Unido) que son naciones catalogadas por el Banco Mundial como desarrolladas.

Según el Banco Mundial (2008, citado por Lemus y Villatoro 2009), la penetración de computadoras en el G7 por cada mil habitantes es casi ocho veces mayor que en América Latina y 13 veces mayor que en El Salvador. El número de usuarios de Internet por cada mil habitantes en América Latina y El Salvador representan una tercera y quinta parte respectivamente, del número de usuarios de Internet por cada mil habitantes en el G7.

El estudio realizado establece que la virtualización de la educación en El Salvador está en los primeros escalones del desarrollo, muy pocas universidades logran ofertar áreas del conocimiento a través de la internet. Las carreras y materias que ya se están impartiendo de manera digital en las universidades son en las áreas de la ciencias empresariales, y la materias varían en naturaleza hay las que son eminentemente teóricas, pero también las prácticas, de varias carreras universitarias.

Dentro de las universidades aún existe la predominancia de la utilización de ambientes de aprendizaje tradicionales (aula), los espacios virtuales de apoyo no todas las universidades los tienen de manera institucional. Las que los tienen son en sitios gratuitos que permiten la colocación de textos de refuerzo para las asignaturas.

La virtualización puede comprender la representación de procesos y objetos asociados a actividades de enseñanza y aprendizaje, investigación, extensión y gestión, así como objetos cuya manipulación permite al usuario realizar diver- sas operaciones a través de Internet, tales como aprender mediante la interacción con cursos electrónicos, inscribirse en un curso, consultar documentos en una biblioteca electrónica, comunicarse, etc. (Castells, 2001a).

Por su parte, Hopenhayn (2003) asegura que los acelerados cambios que impone la sociedad de la información en el ámbito productivo y comunicacional obliga a los nuevos medios (en donde se incluyen formas de educación y medios de comunicación) a una rápida y ágil adaptación para la transmisión de conocimientos, la comunicación a distancia y el uso de información.

Por otra parte, la inclusión de equidad de género en la producción, manejo y divulgación de la información en las universidades salvadoreñas está empezando, es importante reconocer que en algunas de las instituciones académicas realizan esfuerzos para hacer de la información algo más equitativo e igualitario, pese a que no son políticas institucionales, si hay trabajos institucionales en esa vía.

Son pocas las instituciones que tienen institucionalizada la promoción y garantía de la equidad de género en su funcionamiento y entre su personal docente, administrativo y estudiantil. Si bien en dos de las universidades en donde se pudo realizar la entrevista, no existen políticas establecidas institucionalmente, pero si hay iniciativas que buscan sensibilizar en el tema de género, así como crear espacios que garanticen la equidad de género en el quehacer académico y administrativo de las instituciones. En una universidad se encontró que toman la equidad de género como parte de sus valores humanos y cristianos que se promueven como naturaleza de la institución y han colocado el componente de género en los planes de estudio. En otra de las universidades se está elaborando un plan de acción en esa vía y en las demás universidades no existe nada concreto. 
Las iniciativas que existen en las universidades a las que se tuvo acceso, giran en torno a la realización de actividades, tales como conferencias, talleres, charlas, capacitaciones (para estudiantes y docentes). Es evidente que las instituciones que tienen iniciativas claras sobre enfoque de género trabajan en aras de incluir el enfoque de género en el quehacer de la institución.

Ninguna de las universidades regula de alguna manera el uso del lenguaje textual y gráfico en el portal electrónico de la institución, en la comunicación interna y en los trabajos de graduación de sus estudiantes. La información que se publica en las páginas electrónicas de las universidades en El Salvador no tiene ninguna regulación en cuanto al uso del lenguaje textual y de imagen inclusiva. Eso se comprobó en las páginas electrónicas analizadas, en todas se encontraron sintagmas que denotan lenguaje sexista.

La sociedad de la información y del conocimiento se gesta y se afinca en las sociedades actuales, en unas ya completamente desarrollada, en otras, como El Salvador, a penas en surgimiento. Esta sociedad significa la relación de seres humanos, de integrantes de un mismo sistema social, en esa interacción salen a flote los tradicionales problemas de la convivencia humana, como la desigualdad de género. En ese sentido se hace necesario ver la equidad de las construcciones sociales de hombres y mujeres. Si en las sociedades tradicionales, los valores de desigualdad han estado presentes, al transformarse en sociedades de la información y del conocimiento, pues esos valores se han transmitido y están presentes en cada punto de las sociedades modernas, como en la producción, gestión y difusión de la información.
El Observatorio para la Cibersociedad (2004) recomienda la creación de redes de mujeres, y a través de ellas promover la creación de foros abiertos y permanentes sobre TIC y género. En el mundo actual es necesaria la promoción activa de relaciones sinérgicas por la equidad de género y las interrelaciones entre redes de mujeres y redes por la equidad de género.

Web con género (2005) asegura que la sociedad del conocimiento no se está produciendo de manera igualitaria, la complejidad del manejo de los dispositivos que permiten acceder a ella y la necesaria adquisición de nuevos conocimientos provocan variadas y diferentes experiencias personales y originando desigualdades tanto verticales como horizontales en la utilización de las TIC.

En la sociedad de la información y del conocimiento, las páginas Web constituyen un nuevo tipo de producto audiovisual en donde la integración de diferentes lenguajes con igualdad de importancia conjuga mensajes para difundir información. (Valdés, 2008 y Observatorio de la Cibersociedad, 2004). En Iberoamérica, muchas de sus universidades más importantes están evidenciando serias y continuas limitaciones para poder modificar rápida y profundamente sus modelos, estructuras y procedimientos obsoletos, con la finalidad de responder funcional y oportunamente a las nuevas y exigentes demandas (Casas, 2005).

De ahí la importancia de cómo en El Salvador y sus universidades se gestiona el conocimiento, de cómo se transmite la información como generadora de saber, dependiendo de cómo se hace, pues esa es la sociedad de la información y del conocimiento que se está construyendo en el país. 


\section{Referencias}

- Casas, M., 2005, La Nueva Universidad ante la Sociedad del Conocimiento, Revista de Universidad y Sociedad del Conocimiento, Vol 2, UNESCO, ISSN 1698-580X, consultado en http://www.uoc.edu/rusc/2/2/dt/esp/casas.pdf

- Castells, M. (2001a). La era de la información. vol. 1. La sociedad red. Madrid: Alianza Editorial.

- Castells, M. (2001b). La Galaxia Internet. Madrid: Areté.

- Cazaux, D., 2010, La comunicación pública de la ciencia y la tecnología en la sociedad del conocimiento, Revista Palabra y Razón, Organización de Estados Iberoamericanos, número 65. http://www.razonypalabra.org.mx/N/ n65/actual/dcasaux.html

- Cepal, 2005, Los caminos hacia una sociedad de la información en América Latina y el Caribe recuperado de http://www.cepal.cl/publicaciones/xml/9/12899/lcg2195e2.pdf

- Cepal, 2008, La sociedad de la información en América Latina y el Caribe Desarrollo de las tecnologías y tecnologías para el desarrollo 2008, consultado en http://www. oei.es/tic/cepal.pdf

- Cepal/Eclac, 2008, Sociedades de la información en América Latina y el Caribe: Avances y desafíos. http://www.eclac.org/socinfo/noticias/noticias/7/32637/ Colombia.pdf

- Conacyt, 2010, Indicadores de ciencia y tecnología. Estadísticas sobre actividades científico y tecnológicas. Sector de educación superior, Consejo Nacional de Ciencia y Tecnología-departamento de Desarrollo Científico y Tecnológico.

- Fontcuberta, M., 2000, Medios, comunicación humana y sociedad del conocimiento, revista Comunicar número 14, Colectivo Andaluz para la educación en los medios de comunicación, Andalucía España.
- Hilbert, M., Cairó, O., 2009, ¿Quo vadis, tecnología de la información y de las comunicaciones?, Cepal.

- Hopenhayn, M., 2003, Educación, comunicación y cultura, en la sociedad de la información: una perspectiva latinoamericana. Serie Informes y estudios especiales, Cepal, Santiago de Chile. ISBN: 92-1-322130-4.

- Lemus, A., Villatoro, C., 2009, La brecha digital en El Salvador: causas y manifestaciones, trabajo de graduación, Universidad José Simeón Cañas.

- Machlup, F., 1962, The Production and Distribution of Knowledge in the United States.

- Murolo, N., 2010, Cuatro conceptos para interpretar el cruce entre digitalización y sociedad, revista de temas sociales Kairos, http://www.revistakairos.org/k26-archivos/murolo.pdf

- Museo de la información, sf, La sociedad del conocimiento: la digitalización y las redes, http://www.inegi. gob.mx/inegi/contenidos/espanol/ciberhabitat/museo/ sociedad/02.htm.

- Observatorio para la Cibersociedad, 2004, ¿Hacia qué sociedad del conocimiento? II Congreso ON- LINE del Observatorio para la Ciber Sociedad.

http://www.cibersociedad.net/congres2004/index es.html.

- Toffler, A., 1994, El cambio del poder, segunda edición, Plaza \& Janes editores, Madrid, España.

- Unesco, 2005, Hacia las sociedades del conocimiento, Unesco, ISBN 92-3-304000.

- Valdés, C., 2008, The Localization of promotional discourse on the internet, en Proyecto Web con Género. Pág.12,

- Web con Género, 2005, Estudio sobre el lenguaje y contenido sexista en la web, España. http://genero.bvsalud. org/lildbi/docsonline/8/2/1028-ESTUDIO_SOBRE_LENGUAJE.pdf 